\title{
OntoEditor: a Web Tool for Manipulating Ontologies Stored in Database Servers
}

\author{
Karine F. Vasconcelos, Cláudio S. Baptista, Ladjane S. Arruda, Ulrich Schiel \\ Universidade Federal de Campina Grande \\ Departamento de Sistemas e Computação \\ Av. Aprigio Veloso, 882 Bodocongó \\ 58109-970 Campina Grande - Pb Brasil \\ $\{$ karine|baptista|ladjane|ulrich\}@dsc.ufcg.edu.br
}

\begin{abstract}
The Web is moving to a new generation in which machine-understandable processing is mandatory. In order to achieve this goal it is essential to define ontologies which enable the modeling of application domains and can be shared and understood by different applications in different platforms. These ontologies are complex and so it is necessary to provide software tools which aims to facilitate ontology manipulation. In this paper, we describe a new tool for ontology manipulation known as OntoEditor. OntoEditor is a Web tool, which has a graphical interface for representing an ontology graph. Moreover, OntoEditor uses a database management system for ontology persistency and query manipulation. The ontologies are represented internally as RDF and RDF Schema.
\end{abstract}

Keywords: ontology, semantic web, RDF

\section{Introduction}

In the last few years application development has used Web browsers as the default interface. In these applications, the Web has been used for human communication. However, the new wave is to provide machine communication without human interference. For instance, e-commerce applications are examples of this new demand, which has been called semantic [Berners-Lee 1999]. 
In the semantic Web it is fundamental to model different knowledge domains. Hence, ontologies are used with this aim [Decker 2000, Gruber 1993]. In a simple view, an ontology can be defined as a vocabulary with terms and relationships which enable modeling the universe of discourse, or more precisely a specific domain. Ontologies help to achieve semantic interoperability which is essential in machine understanding. An ontology is tipically a graph of concepts and their relationships and properties.

Due to the complexity involved in the creation and manipulation of ontologies, it is necessary to develop software tools to deal with them in a agile and easy way. Several tools have been proposed so far, such as Protégé, OilEd e KAON [Noy 2001, Bechhofer 2001, Handschuh 2001], we discuss them in section 2. In this paper we propose a new tool, called OntoEditor, which uses a graph data structure to visualize the ontologies and provides persistence and query capabilites using a database server.

The rest of this paper is structured as follow: section 2 presents related work; section 3 discusses the OntoEditor conceptual model; section 4 shows the OntoEditor architecture and design issues; section 5 presents na example using the OntoEditor; and finally, section 6 concludes the paper and discusses further work to be undertaken.

\section{Related Work}

Several tools for manipulating ontologies have been proposed recently. In this section we discuss three of them: Protegé, OntoEdit e OilEd. We will detail the main capabilities of each tool and at the end of this section we present the capabilities provided by the OntoEditor which are not supported by these tools.

- Protégé: It has been developed by a research team at the University of Stanford, USA, for more than 15 years. The Protégé Project is one of the precursors as a tool for modeling and building knowledge bases. The Protégé knowledge acquisition model uses a tree for representing term hierarchy. Protégé enables the building of colaborative ontologies with migration, integration and version control capabilities. However, it does not provides persistence facilities for storing and retrieving these ontologies, as only a file system is used for storing the ontologies [Noy 2001]. 
- KAON: The KAON (Karlsruhe Ontology and Semantic Web infrastructure) is an editor which, although it does not have a flexible user interface as Protege, has similar concepts such as ontology building using tree data structure; many representation languages such RDF and DAML+OIL. [Handschuh 2001]. Again, there is no support for database persistency on $\mathrm{KAON}$, although there is a tool called REVERSE which enables the mapping of relational database into ontologies

- OILED: This editor was the first to enable the representation of ontologies using the DAML+OIL language. Although it is based on Protégé, OILED is very simple and does not cope with large ontologies. It can export ontologies in several formats including OIL, OIL-RDFS, DAML-OIL e HTML [Bechhofer 2001].

The OntoEditor has some interesting features that are not provided by the mentioned tools. For example, the three tools (Protégé, KAON e OILED) have a tree interface to represent ontologies, even though a graph struture is the natural representation for ontologies. In this sense, with OntoEditor, an ontology is defined and presented as a graph. Another important feature allowed by OntoEditor is the use of a database system for ontology persistence and retrieval. This feature is not provided by the others tools. Hence, the OntoEditor supports querying the ontologies using metadata and the own ontology description, since it queries RDF using XMLQL query language [REFERÊNCIA]. For instance, an user is looking for ontologies which have a specific term or property (e.g. red wine), then he/she can use the query facility to obtain the ontologies that satisfy her requirements.

\section{OntoEditor: Conceptual Model}

The main aim of OntoEditor is to create and manipulate ontologies with presistence provided by a database system. Ontologies are defined using an abstract model which is based on RDF(Resource Description Framework) [Lassila 1999]. As mentioned before the strucutre is a graph. 
In the OntoEditor, an ontology is composed by the following elements: Terms, Properties and Relationships. Moreover, an ontology contains metadata which describe some properties including:

- Name contains the name given to an ontology. For example: "Genome";

- Author contains the author or responsible for the ontology. For example: "John Smith";

- Domain indicates the main knowledge domain used in the ontology. For example: "Biology";

- Date of Creation contains the date of creation of the ontology. For example: "10/07/1997".

Beside the metadata, to represent an ontology is is necessary to use the following concepts:

- Terms: represent the key-concepts extracted from a given knowledge domain. Each term has attributes such as name (mandatory) and description (non-mandatory). Moreover each term may have properties, which are described below.

- Properties: represent any other attributes which describes a defined term. Each property has attributes such as name, type and value. For example, one can define a property for a term Person as: $<$ name $=$ age, type $=$ integer, value $=30>$. After defining a property, it will be added to a term. Then, this property may be associated to other terms (como assim?). These properties may be updated or deleted at any time.

- Relationships: involve relationships among terms. A term may be related or not to one or more terms. Each relationship has only an attribute which is its name. The tool suggests some relationships to the user, however new relationships can be added. The suggested relationships subclass, synonym, hypernym, and hyponym. These relationships may be updated or deleted.

The OntoEditor conceptual model is presented in figure 1 (está em português) using UML notation [Booch 1998]. The class ontology contains the properties (metadata) of an 
ontology. An ontology is an aggregation of terms, A term can be extended via inheritance (not on the figure).
Excluído: (na figura não foi usado o símbolo da agregação)

Excluído: with their what??

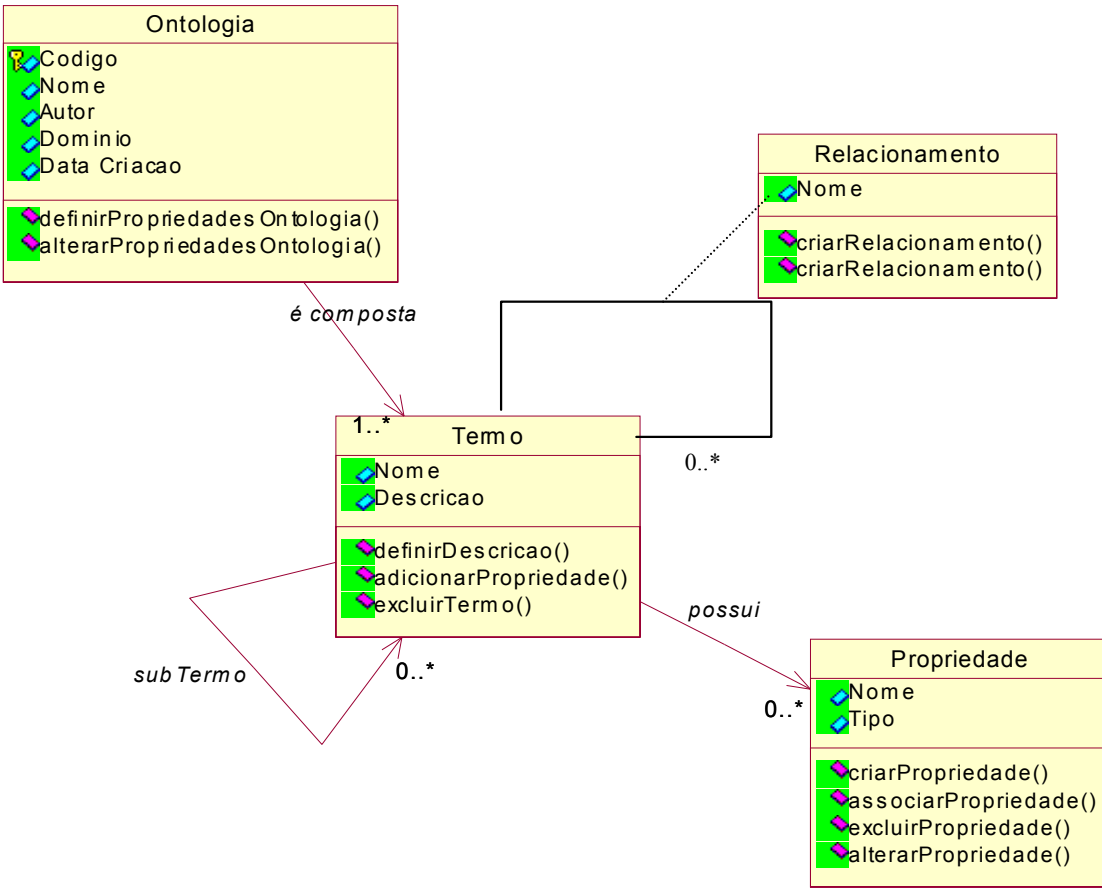

Figure 1. OntoEditor - Conceptual Model

\section{OntoEditor: Design and Implementation Issues}

The design of the OntoEditor tool aims to build a simple and useful ontology editor. Figure 2 presents the overall architecture of the OntoEditor.

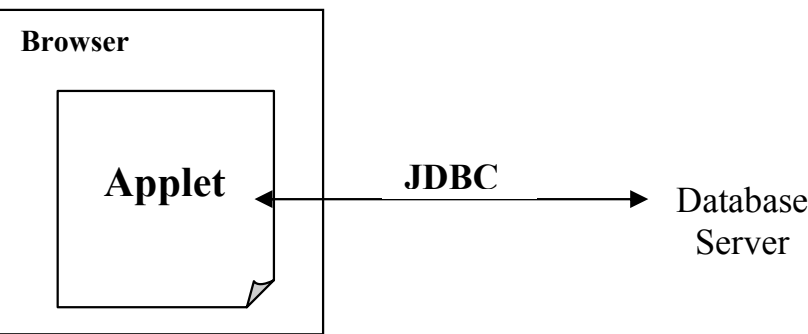


The OntoEditor was implemented as a Java Applet using the client-server model. It has a Web graphical interface using Swing components. This enables graphical manipulation of the ontologies, so that, the user can build and viasualize a given ontology as a graph. After creating an ontology, the user can save it in a relational database system or export it as a text file (in RDF format??).

By using an Applet it is possible to execute the editor from any browser, without requiring any installation process on the client machine. However, it is important to mention that Java Applets have security restrictions, as for example, saving files in the host machine. Fortunately, signatures can be used to give permission for an Applet to execute, including permission to write in the local disk.

The communication with the database server is done using the JDBC API. The database schema has a table called Ontologies with the following attributes: code, name, author, domain, date of creation, and URL. The code represents a surrogate which identifies uniquely an ontology, the attributes name, author, domain and date of creation are metadata attributes which were explained before. Finally, the URL has the URL of a RDF file which contains the ontology contents. This RDF document is generated by the OntoEditor from the graph created by the user.

As it was mentioned previously, the user builds its ontology graphically and this oontology is saved in a database or exported to a file. In order to achieve that, it is necessary to establish a translation process between a graph and RDF and vice-versa.

OntoEditor uses two languages to represent an ontology: RDF and RDF Schema [Brickey 2002]. From now on, when we mention RDF, it means RDF/RDF Schema. There exists an intermediary process which is responsible for translating the graphs into an RDF specification. Figure 3 illustrates this translation process. 


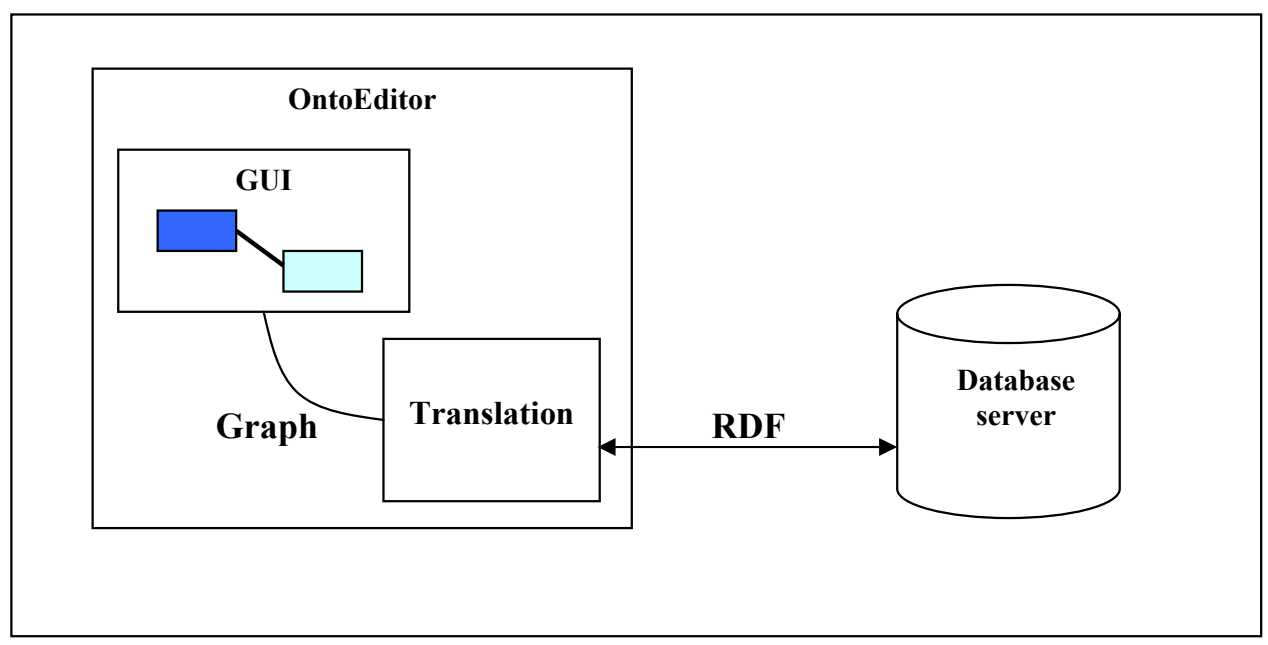

Figure 3. Graph - RDF translation process

During this translation process OntoEditor uses the Jena API for parsing an RDF document [McBride 2000]. The translation process is bi-directional:

- Graph-RDF translation -when a Save or Export or View RDF menu options is chosen, the graph representing an ontology is translated into an RDF document.

- RDF-Graph Translation - occurs when either Open or Import options are chosen. Hence, a graph is displayed from a RDF document.

\subsection{Querying Ontologies}

One of the main capabilities of the OntoEditor is its query system. The OntoEditor provides a query menu in which users may either query or edit stored ontologies. Different queries can be realized including:

1 - Queries on the ontology properties. For instance author, domain, date of creation,

Example1: a user would like to query ontologies in which "John Smith" is one of the authors.

2 - Queries on the ontology contents: in this case the terms, properties and relationships may be retrieved.

Example2: a user would like to query ontologies that talk about "University". 
In this case, a query is mounted using the XMLQL language and submitted to a XMLQL processor [Florescu 1999].

The ontology query process consists basically of:

- First, the system mounts a XMLQL query using user data;

- Second, the XMLQL processor is called;

- Third, for each ontology that contains the searched element, a value is returned for that ontology.

- Last, it accesses the database looking for more information about the retrieved term.

For example, let us suppose that an user would like to search an element called library. The query mounted by the system using XMLQL would be:

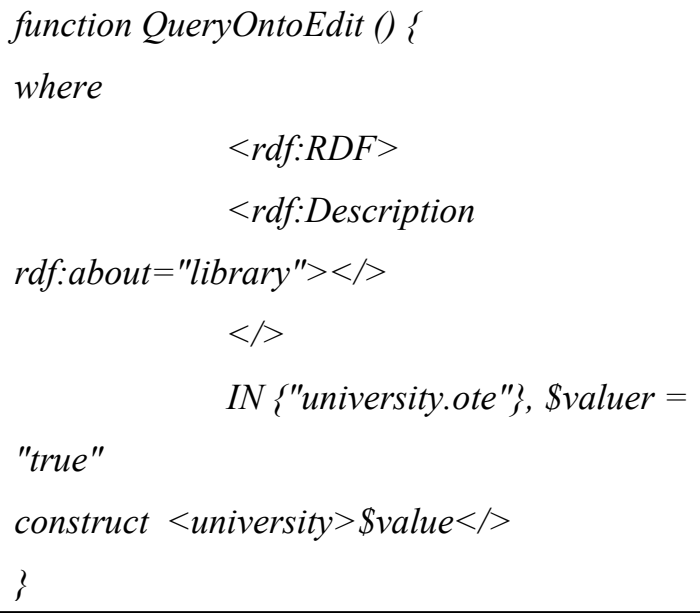

As a result, we would have:

$<? x m l$ version $=" 1.0$ " encoding $=" U T F-8$ "? $>$

$<$ university $>$ true $<$ /university $>$

This result means that in the University Ontology there is an element library.. 


\section{An Example}

In this section we present an example of building an ontology form the University domain.

The ontology will be composed by a set of terms semantically related, with its attributes, properties and relationships. In this example the University ontology should have

- a place name, such as city, state, country;

- a set of courses. These courses alocate many people which could be University employees or students. Moreover, these people may have publications such as thesis, papers, and books;

- professors and assistants as employees;

- undergradutes and graduates. $\mathrm{PhD}$ students may be assistants.

Figure 4 presents the specified University ontology. The user may translate this graph to a RDF file which can be stored in a database server.

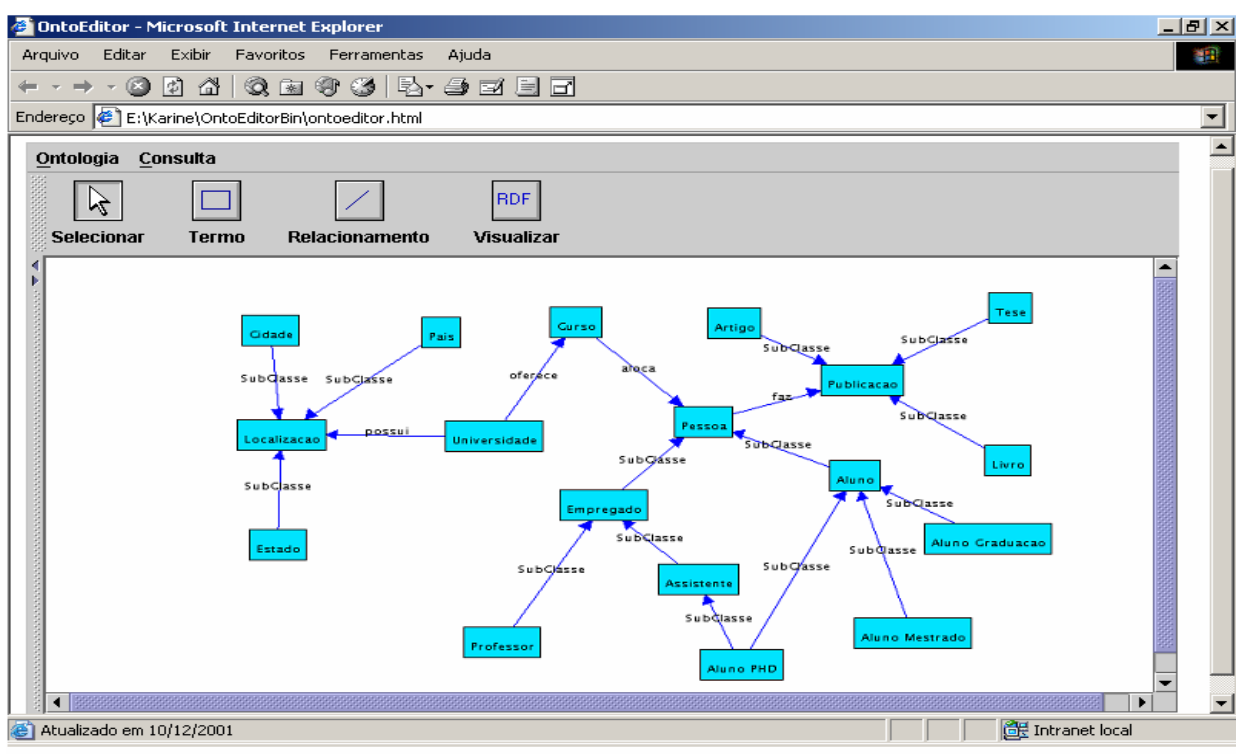

Figure 4. Building the University Ontology 


\section{Conclusion}

In this paper we have presented a software tool called OntoEditor which enables the creation, edition, update, storing and querying of ontologies. The OntoEditor permits to represent an ontology as a graph, which increases its usability. Moreover, it uses the capabilities provided by a database server to store and query several ontologies using metadata, and the own ontologies can be queried using XMLQL.

As further work, we intend to incorporate OWL as an alternative for representing the ontologies and make use of XQuery as the query language. Moreover, we intend to translate ontologies from RDF to OWL and vice-versa.

Since ontologies are conceptually related together by means of sub-ontologies, overlapping, and so on, these relationships between ontologies must be considered in a really powerful tool for the complexity of the Semantic Web.

\section{References}

[Bechhofer 2001] Sean Bechhofer, Ian Horrocks, Carole Goble e Robert Stevens. OilEd: a Reason-able Ontology Editor for the Semantic Web. Proceedings of KI2001, Joint German/Austrian Conference on Artificial Intelligence. Lecture Notes in Computer Science 2174, Springer-Verlag, Páginas 396-408, 2001.

[Berners-Lee 1999] T. Berners-Lee, Weaving the Web, Harper, San Francisco, 1999

[Booch 1998] Grady Booch, Ivar Jacobson, James Rumbaugh. The Unified Modeling Language User Guide. Addison-Wesley, 1998.

[Brickley 2002] Dan Brickley e R.V. Guha (eds) RDF Vocabulary Description Language 1.0: RDF Schema, http://www.w3.org/TR/rdf-schema/ 
[Decker 2000] Stefan Decker, Frank van Harmelen, Jeen Broekstra, Michael Erdmann, Dieter Fensel, Ian Horrocks, Michel Klein, Sergey Melnik: The Semantic Web - on the Roles of XML and RDF . In: IEEE Internet Computing. September/October 2000.

[Florescu 1999] D. Florescu, A. Deutsch, A. Levy, D. Suciu, M. Fernandez, A Query Language for $\{X M L\}$ In Proceedings of Eighth International World Wide Web Conference, 1999

[Gruber 1993] T. Gruber. A Translation Approach to Portable Ontology Specifications. Knowledge Acquisition, 5(2):199-220, 1993.

[Handschuh 2001] Siegfried Handschuh, Alexander Maedche, Ljiljana Stojanovic e Raphael Volz. KAON - The KArlsruhe ONtology and Semantic Web Infrastructure 2001.

[Lassila 1999] O. Lasilla, R. Swick (eds): Resource Description Framework (RDF) Model and Syntax Specification, http://www.w3.org/TR/REC-rdf-syntax/.

[McBride 2000] Brian McBride. Jena: Implementing the RDF Model and Syntax Specification. http://www-uk.hpl.hp.com/people/bwm/papers/20001221-paper.

[Noy et al. 2001] Natalya F. Noy, Michael Sintek, Stefan Decker, Mônica Crubézy, Ray W. Fergerson e Mark A. Musen. Creating Semantic Web Contents with Protege2000 Intelligent Systems 16(2):60-71, 2001. 